\title{
SPEM: A state-of-the-art instrument for high resolution molecular imaging of small animal organs
}

\author{
SPEM: 0 estado da arte em instrumentação para imagem molecular \\ de alta resolução de órgãos de pequenos animais
}

Marilia Alves dos Reis ${ }^{1}$, Jorge Mejia ${ }^{2}$, Ilza Rosa Batista ${ }^{1}$, Marycel Rosa Felisa Figols de Barboza ${ }^{3}$, Solange Amorim Nogueira ${ }^{4}$, Jairo Wagner ${ }^{4}$, Francisco Romero Cabral ${ }^{5}$, Petrick Marcellus Victorio Mendonça Davoglio ${ }^{6}$, Vanessa Costhek Abílio7, Geng Fu ${ }^{8}$, Nan Li ${ }^{8}$, Ling Jian Meng ${ }^{9}$, Ming Chi Shih ${ }^{10}$, Chin-Tu Chen ${ }^{11}$,

Edson Amaro Junior ${ }^{3}$, Rodrigo Affonseca Bressan ${ }^{12}$

\begin{abstract}
Objective: To describe the Single Photon Emission Microscope (SPEM), a state-of-the-art instrument for small animal SPECT imaging, and characterize its performance presenting typical images of different animal organs. Methods: SPEM consists of two independent imaging devices based on high resolution scintillators, high sensitivity and resolution Electron-Multiplying CCDs and multi-pinhole collimators. During image acquisition, the mouse is placed in a rotational vertical holder between the imaging devices. Subsequently, an appropriate software tool based on the Maximum Likelihood algorithm iteratively produces the volumetric image. Radiopharmaceuticals for imaging kidneys, heart, thyroid and brain were used. The mice were injected with 74 to $148 \mathrm{MBq} / 0,3 \mathrm{~mL}$ and scanned for 40 to 80 minutes, 30 to 60 minutes afterwards. During this procedure, the animals remained under ketamine/xilazine anesthesia. Results: SPEM images of different mouse organs are presented, attesting the imaging
\end{abstract}

capabilities of the instrument. Conclusion: SPEM is an innovative technology for small animal SPECT imaging providing high resolution images with appropriate sensitivity for pre-clinical research. Its use with appropriate radiotracers will allow translational investigation of several animal models of human diseases, their pharmacological treatment and the development of potential new therapeutic agents.

Keywords: Molecular imaging; Pre-clinical research; Small animals; Single photon emission computed tomography; Multipinhole collimation

\section{RESUMO}

Objetivo: Descrever o Single Photon Emission Microscope (SPEM), o estado da arte em tecnologia para SPECT de pequenos animais, e ilustrar seu desempenho com imagens típicas de diferentes órgãos de animais. Métodos: 0 SPEM consiste em dois dispositivos de

\footnotetext{
Study carried out at Centro de Imagem Pré-Clínica, Centro de Experimentação e Treinamento em Cirurgia of the Instituto Israelita de Ensino e Pesquisa Albert Einstein - IIEPAE, São Paulo (SP), Brazil. 1 Instituto do Cérebro - InCe, Hospital Israelita Albert Einstein - HIAE, São Paulo (SP), Brazil; Laboratório Interdisciplinar de Neurociências Clínicas - LiNC, Universidade Federal de São Paulo - UNIFESP, São Paulo (SP), Brazil.

${ }^{2}$ Instituto do Cérebro - InCe, Hospital Israelita Albert Einstein - HIAE, São Paulo (SP), Brazil; Faculdade de Medicina de São José do Rio Preto - FAMERP - São José do Rio Preto (SP), Brazil.

${ }^{3}$ Department of Diagnostic Imaging and Instituto do Cérebro - InCe, Hospital Israelita Albert Einstein - HIAE, São Paulo (SP), Brazil.

${ }^{4}$ Department of Diagnostic Imaging, Hospital Israelita Albert Einstein - HIAE, São Paulo (SP), Brazil.

${ }^{5}$ Instituto do Cérebro - InCe, Hospital Israelita Albert Einstein - HIAE, São Paulo (SP), Brazil.

${ }^{6}$ Department of Clinical Engineering, Hospital Israelita Albert Einstein - HIAE, São Paulo (SP), Brazil.

7 Department of Pharmacology and Laboratório Interdisciplinar de Neurociências Clínicas - LiNC, Universidade Federal de São Paulo - UNIFESP, São Paulo (SP), Brazil.

${ }^{8}$ University of Illinois at Urbana-Champaign, University of Illinois, Urbana-Champaign (IL), Estados Unidos.

${ }^{9}$ Department of Bioenginnering at the University of Illinois at Urbana-Champaign, Urbana-Champaign (IL), Estados Unidos.

${ }^{10}$ Instituto do Cérebro - InCe, Hospital Israelita Albert Einstein - HIAE, São Paulo (SP), Brazil; University of Chicago (IL), Estados Unidos.

${ }^{11}$ Committee on Medical Physics and the College at University of Chicago, Chicago (IL), Estados Unidos.

${ }^{12}$ Department of Psychiatry of the Universidade Federal de São Paulo - UNIFESP, São Paulo (SP), Brazil; Laboratório Interdisciplinar de Neurociências Clínicas - LiNC, Universidade Federal de São Paulo UNIFESP, São Paulo (SP), Brazil; Instituto do Cérebro - InCe, Hospital Israelita Albert Einstein - HIAE, São Paulo (SP), Brazil.

Corresponding author: Marília Alves dos Reis - Instituto do Cérebro - Instituto Israelita de Ensino e Pesquisa Albert Einstein (IIEPAE) - Avenida Albert Einstein, 627/701 - Morumbi - Zip code: 05652-900 São Paulo (SP), Brazil - Phone: (55 11) 2151-3727 - E-mail: bol013@einstein.br
}

Received on: Mar 19, 2012 - Accepted on: May 7, 2012

Conflict of interest: none. 
imagem independentes que usam cintiladores de alta resolução, câmaras CCD de altas sensibilidade e resolução, e colimadores multiorifício. Durante a aquisição das imagens, o camundongo é posicionado dentro de um tubo vertical rotatório entre os dispositivos imageadores. Posteriormente, um software apropriado, baseado no algoritmo de Máxima Verossimilhança, produz iterativamente a imagem tomográfica. Utilizamos radiofármacos apropriados para imageamento de rins, tireoide, coração e cérebro. Os camundongos foram injetados com 74-148 MBq/0,3mL e foram registrados por 40 a 80 minutos, entre 30 a 60 minutos pós-injeção. Durante esse processo, os animais permaneceram anestesiados com quetamina/ xilazina. Resultados: Apresentamos imagens tomográficas de diferentes órgãos de camundongos, que indicam a capacidade do instrumento na aplicação proposta. Conclusão: 0 SPEM é uma tecnologia inovadora de SPECT para pequenos animais, que fornece imagens de alta resolução com sensibilidade apropriada para estudos pré-clínicos. Seu uso com radiofármacos apropriados permitirá a realização de estudos translacionais de vários modelos animais de doenças humanas e seu tratamento farmacológico, bem como 0 desenvolvimento de novos agentes terapêuticos.

Descritores: Imagem molecular; Pesquisa pré-clínica; Pequenos animais; Tomografia computadorizada por emissão de fóton único; Colimadores multiorifício

\section{INTRODUCTION}

In the clinical environment, in vivo imaging techniques are considered standard tools used to visualize internal organs and tissues inside the human body. Techniques like ultrasound (US), computed tomography (CT), magnetic resonance imaging (MRI), single photon emission computed tomography (SPECT) and positron emission tomography (PET) allow the study of morphology or functionality, in normal or pathological conditions.

In the last two decades, a great effort has been done to transpose molecular imaging techniques to the pre-clinical environment, in which small laboratory animals (mostly rats and mice) are routinely used. However, as the small animal organs can be at least 300 times smaller in volume than human ones, two main challenges are faced, which hamper the direct application of those techniques: how to obtain appropriate spatial resolution and sensitivity to image them $^{(1)}$ ?

In the specific case of clinical SPECT, planar projections are acquired using parallel hole collimators and are combined by means of the Filtered Backprojection algorithm to produce volumetric reconstructions ${ }^{(2)}$. This procedure limits the spatial resolution to something around $7 \mathrm{~mm}^{(3)}$. If applied to small animals, this value is clearly unacceptable. Pinhole and multi-pinhole based collimators have being proposed as an alternative to the parallel hole collimators ${ }^{(4)}$. In combination with scintillator or semiconductor detectors, adaptations of clinical instruments or special purpose devices are under development ${ }^{(5)}$. Given the magnification factor inherent to pinhole collimators, images of small structures can be largely amplified, allowing the visualization of very small features. Visualization of details is also dependent on the size of the collimator aperture: the smaller the aperture, the better the spatial resolution. However, reducing the size of the aperture limits the quantity of radiation that can be registered by the imaging instrument. Thus, there is a trade-off between spatial resolution and sensitivity. To solve this challenge, collimators with appropriately distributed, multiple pinholes, in combination with specifically developed software tools, allow the increase of sensitivity without sacrificing spatial resolution.

An additional challenge is faced when imaging small animal organs: given the reduced size of the targets, even minute misalignments or vibrations introduced by rotating the detector and collimator around the animal may prevent that good quality images can be obtained. As a solution, it has been proposed to rotate the animal in front of the imaging instrument instead of the instrument around the target $^{(6)}$. In the most extreme situation, neither the animal nor the instrument are rotated, in which case a set of detectors is mounted completely surrounding the animal under study ${ }^{(7,8)}$.

On the other hand, SPECT allows imaging animal organs with radiopharmaceuticals labeled with radioactive elements emitting at different energies, for example, metastable Technetium- $99\left({ }^{99 \mathrm{~m}} \mathrm{Tc}\right)$, Iodine- 123 $\left({ }^{123} \mathrm{I}\right)$, Thallium-201 $\left({ }^{201} \mathrm{Tl}\right)$ and Indium-111 $\left({ }^{111} \mathrm{In}\right)$. In that way, at least in principle, evaluation of different processes or organs can be done simultaneously. In addition, radiopharmaceuticals for SPECT use to have longer mean lives, if compared to PET ones, given the opportunity to study slower processes or the evolution of the same process at different point times.

In the recent past, the SPEM instrument was installed in our imaging facility, opening the great opportunity to develop molecular imaging research in animal models of human diseases. In this article, we present the Single Photon Emission Microscope (SPEM), a state-of-the-art molecular imaging SPECT instrument for small animals. Based on multi-pinhole collimation, high resolution scintillators and high sensitivity recording devices, this equipment allows obtaining functional tomographic images of small 
animal organs under the most diverse experimental conditions. The performance of the instrument is illustrated by means of SPECT images of different mice organs.

\section{OBJECTIVES}

To describe the Single Photon Emission Microscope (SPEM), a state-of-the-art instrument for small animal SPECT imaging, and characterize its performance presenting typical images.

\section{METHODS}

SPEM (Figure 1) is an instrument designed by the Department of Radiology and Medical Physics Committee of the University of Chicago to obtain SPECT images of small volume targets ${ }^{(9-11)}$. In May 2010, this instrument was installed at the Centro de Imagem Pré-Clínica of Centro de Experimentação $e$ Treinamento em Cirurgia (CETEC) of Instituto Israelita de Ensino e Pesquisa Albert Einstein (IIEPAE) as a collaborative endeavor, with the main objective of developing strategies and experimental protocols for its characterization as an imaging instrument to be used in the pre-clinical research environment.

The instrument consists of two independent imaging devices based on high spatial resolution columnar scintillators, in combination with an image intensifier and high sensitivity, high resolution Electron Multiplying Charge-Coupled Device (EMCCD) cameras. To guarantee high spatial resolution and sensitivity, the imaging devices use 7- and 19-pinhole collimators with



Figure 1. The SPEM pre-clicnical SPECT imaging instrument. The main parts of the instrument are indicated
300 and $450 \mu \mathrm{m}$ in diameter, respectively. During the image acquisition procedure, the animal is placed in a vertical holder which rotates in front of the imaging detector, in that way minimizing the oscillation of the internal organs. After acquisition of the planar projections, a specially designed software tool based on the Maximum Likelihood algorithm ${ }^{(12)}$ iteratively searches for the model of the radiopharmaceutical distribution on the target that better explains the recorded projections, considering the physics of the imaging procedure.

With those characteristics, the SPEM instrument can reach a tomographic spatial resolution between 150 and $350 \mu \mathrm{m}$, when imaging the ${ }^{125} \mathrm{I}(25-30 \mathrm{keV})$ or the ${ }^{99 \mathrm{~m}} \mathrm{Tc}(140 \mathrm{keV})$ gamma emission ${ }^{(13)}$. With this resolution, tiny sub-compartments inside the mouse's brain can be visualized. Additionally, a CT system can be fixed to the SPECT device, which allows simultaneous co-registering of anatomical and functional information.

\section{Image acquisition with SPEM}

The image acquisition protocol consists of registering a double sequence of planar projections, with each one of the individual imaging devices. This procedure is based on what is called "photon-counting" strategy. Gamma-ray photons are emitted by the target, which arrive to the detector, and interact with it, producing a localized flash of visible light. This light is amplified by the image intensifier and projected on the EMCCD sensor. The CCD cameras register frames at very high speed (typically 50 frames per second) such that individual frames contain just a reduced number of photonic events. Large sets of frames are registered corresponding to the integration time per projection. After acquisition of these raw frames for the complete imaging protocol, the files are analyzed to identify the individual photonic events as the pixels in an 11x11element window around the maximum intensity image elements. With those data, list-mode data files are created containing the following information: $x$ and $y$ position on the CCD detector of the maximum intensity pixel of each photonic event, $\mathrm{x}$ and $\mathrm{y}$ position on the CCD detector of the centroid of the intensity distribution of each photonic event, total intensity of the photonic event and projection to which it corresponds. Having this information, the planar projections are created, as the overlapping of all the events per projection on individual $256 \times 256-$ or $512 \times 512$-element arrays, given their energy is between reasonable limits for the corresponding radioisotope. 
To illustrate the performance of the SPEM, images of four different mice organs were obtained (Figures 2 to 6 ). In all the cases, 3-month old male Swiss mice (nearly $30 \mathrm{~g}$ ) were studied. The radiopharmaceuticals were always injected in the tail vein, and appropriate washout time was considered for each protocol to optimize the target to background intensity ratio. During the imaging procedure, the animals were anesthetized with a ketamine/xilazine combination. Table 1 summarizes specific details of each imaging protocol. For all the image acquisitions, 16 projections were considered.

Considering the size of the target, the number of pinholes in the collimator and the magnification factor of the imaging devices, some overlapping of the individual images can occur. In figure 2, an illustrative
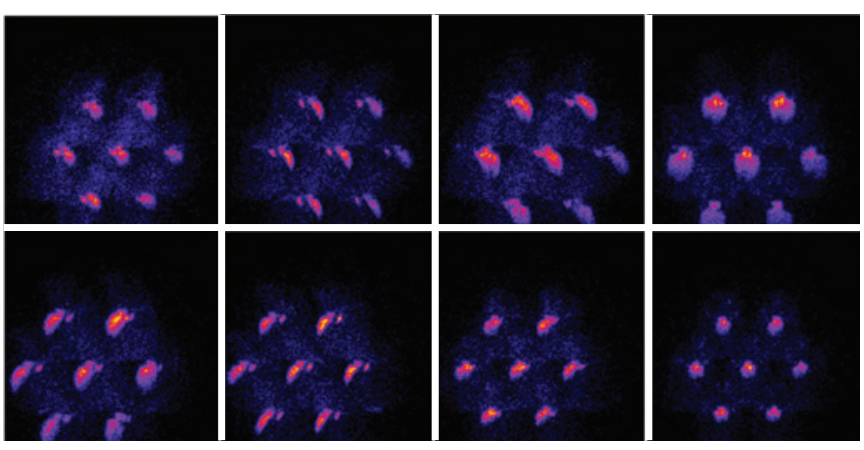

Figure 2. Thyroid gland imaging. Eight out of the 16 projections of the thyroid, acquired with the 7-pinhole collimated device. The larger structure visualized in the images corresponds to the salivary glands
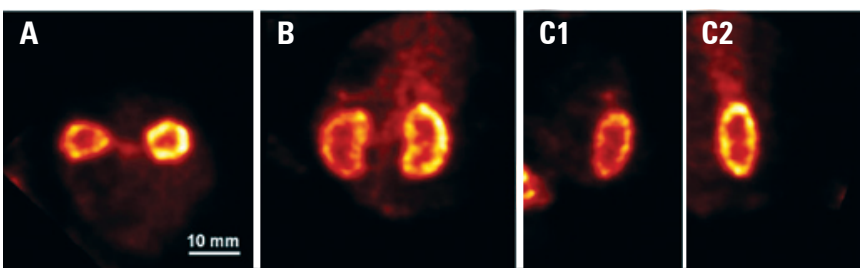

Figure 3. Mouse kidney scan. Intensity slices through the volumetric reconstruction of the mouse kidneys, labeled with $\left[{ }^{99 \mathrm{~m} T C}\right] \mathrm{DMSA}:(\mathrm{A})$ transverse plane; (B) coronal plane; and (C1 and $\mathrm{C} 2$ ) sagittal plane
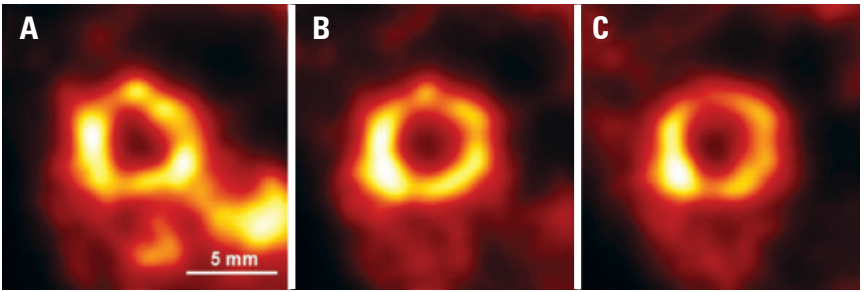

Figure 4. Mouse heart scan. Intensity slices perpendicular to the long axis of the volumetric reconstruction of the mouse heart, labeled with [ $\left.{ }^{99 \mathrm{~m}} \mathrm{Tc}\right]$ Sestamibi. Appropriate reconstruction of the heart was hampered by the signal contribution of neighbor organs, as the liver and kidneys, which overlapped heart images from neighbor pinholes on the individual projections


Figure 5. Mouse thyroid scan. Intensity slices through the volumetric reconstruction of the mouse thyroid, labeled with [ ${ }^{99 \mathrm{~m} T c]}$ Pertechnetate: (A) coronal plane; $(B)$ sagittal plane; and $(C)$ transverse plane. Considering the small size of this organ, this image gives us a hint on the spatial resolution achievable by SPEM during in vivo imaging of animal organs
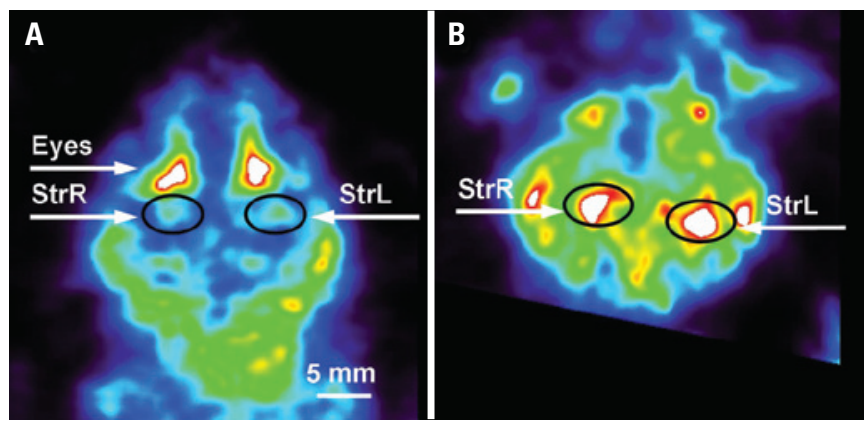

Figure 6. Mouse brain scan. Intensity slices through the volumetric reconstruction of the mouse brain, labeled with [ ${ }^{99 \mathrm{~m} T C}$ ]TRODAT-1: A) transversal brain image; (B) coronal brain image at the level of the Striatum. StrL - Left Striatum; StrR - Rigth Striatum

Table 1. Imaging protocols with SPEM

\begin{tabular}{|c|c|c|c|c|}
\hline Organ & Radiopharmaceutical & $\begin{array}{c}\text { Dose } \\
\text { (MBq) }\end{array}$ & $\begin{array}{c}\text { Washout } \\
\text { time } \\
\text { (min) }\end{array}$ & $\begin{array}{c}\text { Time per } \\
\text { projection } \\
\text { (min) }\end{array}$ \\
\hline Kidneys & $\begin{array}{c}{\left[{ }^{99 m T} \mathrm{~T}\right] \mathrm{DMSA}:} \\
\text { Dimercaptosuccinic acid }\end{array}$ & 74 & 30 & 5 \\
\hline Heart & {$\left[{ }^{99 m} T c\right]$ Sestamibi } & 74 & 60 & 5 \\
\hline Thyroid & {$\left[{ }^{99 m} \mathrm{Tc}\right]$ Sodium Pertechnetate } & 74 & 5 & 3 \\
\hline Brain & [99mTc]TRODAT-1 & 148 & 60 & 5 \\
\hline
\end{tabular}

set of projections is shown, in which the thyroid glands are clearly identifiable. The projections correspond to the 7-pinhole-collimated device.

\section{Volumetric image reconstruction}

To produce volumetric reconstructions, a specially designed software tool, based on the Maximum Likelihood algorithm, is used. This tool searches iteratively for the model of the radiopharmaceutical distribution in the target that better explains the acquired data, considering the statistical nature of the gamma-ray detection process and the physics of the imaging procedure. The reconstruction procedure 
begins with an arbitrary model of the intensity distribution on the target, usually a uniform distribution with all the voxels set to the same unitary value. Given the response of the instrument to a point source (Source Response Function, SRF), that model of the target is projected to the image space to produce a set of model projections. By comparing them with the real projections, a set of error factors is created, which are retroprojected to the object space and combined to be transformed into updating factors. These factors are applied to the original object's radiopharmaceutical distribution model, to create an updated version of it, which is used to re-start all the process. This procedure is repeated iteratively after some finishing criteria are reached. Typically, the process is stopped after reaching an appropriate number of iterations, when there is no noticeable difference between successive resulting images, as evaluated by experienced practitioners. As the number of iterations increases, this algorithm tends to produce noisy images. To avoid that, a smoothing kernel is applied after updating of the object's model. In the current SPEM configuration, a Gaussian filter of variable width is applied, and whose value is modified from 5 to 1 voxels, 1 voxel after every 20 iterations.

\section{RESULTS}

We illustrate the performance of the instrument by presenting the resulting images of four different mouse organs. In all the cases, the images correspond to the projections acquired with the 7-pinhole-collimated device, processed until iteration 80. It is important to highlight that, in some cases, appropriate volumetric image reconstruction is hampered by the overlapping of the planar images projected by the individual pinholes on the same projection. But also, the overlapping signal from organs other than the target ones on neighbor images can difficult to obtain good quality images. In figures 3 to 6 , representative images of different mice organs are shown.

\section{DISCUSSION}

The main purpose of this study was to describe the main characteristics and to show the performance of the SPEM. This new technology SPECT imaging device was developed with the primary objective of obtaining images of small animal organs. Imaging procedure relies on the fact that multiple images can be obtained simultaneously, by using multi-pinhole collimators. However, depending on the size of the emitting volume in the target, the magnification factor and the number of pinholes, individual images can overlap to each other. For this reason, the field of view must be limited, such that image overlapping is minimized or avoided. In its current configuration, the instrument is optimized to image mouse brain structures, even though images of other organs can also be obtained. An appropriate upgrading tool to image larger animals (such as rats and marmosets) is currently under development.

High spatial resolution is the main characteristic of SPEM. Spatial resolution is a function of the intrinsic resolution of the detector, the size of the pinholes on the collimator, the energy of the radiopharmaceutical and the magnification factor. When imaging mouse size targets with ${ }^{125} \mathrm{I}$ labeled radiopharmaceuticals, a spatial resolution of roughly $250 \mu \mathrm{m}$ can be reached. When ${ }^{99 \mathrm{~m}} \mathrm{Tc}$ labeled radiopharmaceuticals are used, a spatial resolution of around $400 \mu \mathrm{m}$ is expected. However, sensitivity is an inverse function of the open area in the collimator. So, reducing the size of the pinholes to improve spatial resolution also decreases sensitivity and, with it, the statistical quality of the resulting images. In order to overcome this condition, the use of multi-pinhole collimators, in combination with appropriate decoding algorithms, have been proposed. In this case, the quality of the images depends on the fraction of overlapping between the images produced by individual pinholes. SPEM uses two independent imaging devices, with 7- and 19-pinhole collimators, with 300 and $450 \mu \mathrm{m}$ in diameter, which make it possible to have simultaneously high spatial resolution and appropriate sensitivity to image mouse organs. By imaging the thyroid glands, we showed that very small targets can be imaged with exquisite quality, provided that the non-overlapping condition mentioned above is satisfied. Even with a limited fraction of overlapping, appropriate details can be visualized when imaging organs such as the heart or kidneys.

Imaging in vivo of the brain in small animals with SPECT or PET remains one of the most challenging applications of the modality because of the small size of brain structures, relatively low tracer uptake, and complex kinetics. By imaging the dopamine transporter with [ $\left.{ }^{99 \mathrm{~m}} \mathrm{Tc}\right]$ TRODAT-1, we showed that this equipment enables to identify small structures like basal ganglia, confirming that this new tool, associated to appropriate radiopharmaceuticals, opens a new window for preclinical research in Neurology.

In order to exemplify and compare the main characteristics of the SPEM instrument, here we 
describe some alternative instruments for small animal SPECT imaging.

MiniSPECT ${ }^{(14)}$ is a low cost upgrading kit for a clinical gamma-camera. In the hardware aspect, it consists of a platform for shield and collimator support, as well as a system for positioning and rotation of the animal in front of the collimator. This platform can be fixed to the head of the clinical SPECT instrument or mounted on an independent cart for easy positioning. In the software aspect, a computer tool based on the Maximum Likelihood algorithm allows for an iterative reconstruction of the emission model in the target. The system can be used with single, double or triple pinhole collimator, depending on the size of the target and the detector. Tomographic spatial resolution of $1.5 \mathrm{~mm}$ can be reached when imaging rat's organs with ${ }^{99 \mathrm{~m}} \mathrm{Tc}$ labeled radiopharmaceuticals. The acquisition and processing time is smaller than 40 minutes per animal, when single pinhole collimator is used.

U-SPECT-II ${ }^{(8)}$ is an instrument based on three large area $\mathrm{NaI}(\mathrm{Tl})$ scintillator detectors mounted in a triangular configuration, in such a way as to completely surround the animal under study. The collimator is made on interchangeable tungsten tubes, which have 75 gold pinhole inserts, all of them oriented toward the same small field of view. Motorized fine movement along the three axes allows the positioning of the organ-of-interest, while monitoring by means of optical CCD cameras. In this instrument, the animal and the detectors stay stationary and 75 different projections are registered simultaneously. With this instrument, spatial resolution better than $0.35 \mathrm{~mm}$ for mouse-size targets or better than $0.8 \mathrm{~mm}$ for rat-size ones has been reported, when imaging ${ }^{99 \mathrm{~m}} \mathrm{Tc}$ labeled radiopharmaceuticals.

NanoSPECT ${ }^{(15)}$ is another instrument based on a set of four scintillator detectors surrounding the target. Each detector is equipped with a multi-pinhole collimator, for a total of up to 64 apertures. The target is maintained stationary during the acquisition, but it is also possible to translate it along the axial direction, while the set of detectors rotates, carrying it out a helical scan for whole body records. With pinholes of 1.0 and $1.4 \mathrm{~mm}$, spatial resolutions of 0.8 and $0.9 \mathrm{~mm}$ for ${ }^{99 \mathrm{~m}} \mathrm{Tc}$ and $1.1 \mathrm{~mm}$ for ${ }^{125} \mathrm{I}$ have been reported.

\section{CONCLUSION}

SPEM is an innovative technology for small animal SPECT imaging, which provides high resolution images with appropriate sensitivity for pre-clinical research. By using the corresponding radiopharmaceuticals, different organs of mice can be finely imaged. Depending on the imaging parameters and the characteristics of the target, tiny mouse organs or small sub-compartments can be evaluated. With simple modifications, mainly related to the magnification factor, larger animals can also be studied, such as rats and marmosets. All the studies we have carried out until this moment were based on ${ }^{99 \mathrm{~m}} \mathrm{Tc}$ labeled radiopharmaceuticals. However, the system is at least equally efficient to detect low energy gamma rays, such as those emitted by ${ }^{125} \mathrm{I}$, with an additional improvement in spatial resolution. Definitely, this kind of technology opens an important window for in vivo studies of the most diverse animal models of human diseases, with special application for neuropsychiatric disorders, such as epilepsy, Alzheimer's disease, Parkinson's disease, depression and schizophrenia. This new tool will enable us to carry out translational research and will provide great support to advance on neuroimaging and in experimental neurology research.

\section{ACKNOWLEDGEMENT}

This work was supported by Associação Beneficente Alzira Denise Hertzog da Silva (ABADHS) and Fundação de Amparo à Pesquisa do Estado de São Paulo (FAPESP): ABADHS's grant FAPESP number 06/61333-3 and FAPESP number 2008/08902-5, respectively.

\section{REFERENCES}

1. Meikle SR, Kench P, Kassiou M, Banati RB. Small animal SPECT and its place in the matrix of molecular imaging technologies. Phys Med Biol. 2005; 50(22):R45-61

2. Cherry SR, Sorenson JA, Phelps ME. Physics in nuclear medicine. 3rd ed Philadelphia: Elsevier Science; 2003.

3. Holly TA, Abbott BG, Al-Mallah M, Calnon DA, Cohen MC, DiFilippo FP, Ficaro EP, Freeman MR, Hendel RC, Jain D, Leonard SM, Nichols KJ, Polk DM, Soman P; American Society of Nuclear Cardiology. Single photon-emission computed tomography. J Nucl Cardiol. 2010;17(5):941-73.

4. Beekman F, van der Have F. The pinhole: gateway to ultra-high-resolution three-dimensional radionuclide imaging. Eur J Nucl Med Mol Imaging. 2007; 34(2):151-61.

5. de Kemp RA, Epstein FH, Catana C, Tsui BM, Ritman EL. Small-animal molecular imaging methods. J Nucl Med. 2010;51(5):18S-32S.

6. Habraken JB, de Bruin K, Shehata M, Booij J, Bennink R, van Eck Smit BL, et al. Evaluation of high-resolution pinhole SPECT using a small rotating animal. J Nucl Med. 2001;42(12):1863-9.

7. Miller BW, Furenlid LR, Moore SK, Barber HB, Nagarkar W, Barrett HH System integration of FastSPECT III, a dedicated SPECT rodent-brain imager based on BazookaSPECT detector technology. IEEE Nucl Sci Symp Conf Rec. 2009:4004-8

8. van der Have F, Vastenhouw B, Ramakers RM, Branderhorst W, Krah JO, Ji C, et al. U-SPECT-II: an ultra-high-resolution device for molecular small-anima imaging. J Nucl Med. 2009;50(4):599-605. 
9. Meng LJ. An intensified EMCCD camera for low energy gamma ray imaging applications. IEEE Trans Nucl Sci. 2006;53(4):2376-84.

10. Meng LJ, Fu G, Roy EJ, Suppe B, Chen CT. An ultrahigh resolution SPECT system for I-125 mouse brain imaging studies. Nuc Inst Meth Phys Res A. 2009;600(2):498-505.

11. Meng LJ, Clinthorne NH, Skinner S, Hay RV, Gross M. Design and feasibility study of a single photon emission microscope system for small animal I-125 imaging. IEEE Trans Nucl Sci. 2006;53(3):1168-78.

12. Shepp LA, Vardi Y. Maximum likelihood reconstruction for emission tomography. IEEE Trans Med Imaging. 1982;1(2):113-22.
13. Fu G, Tan JW, Meng LJ. Aperture design for ultra-high resolution SPECT systems for small animal imaging. IEEE Nucl Sci Symp Conf Rec. 2007;4: 2785-90

14. Mejia J, Galvis-Alonso OY, de Castro AA, Braga J, Leite JP, Simões MV. A clinical gamma camera-based pinhole collimated system for high resolution small animal SPECT imaging. Braz J Med Biol Res. 2010;43(12): 1160-6.

15. Schramm NU, Lackas C, Hoppin JW, Forrer F, de Jong M. The nanoSPECT/ CT: a high-sensitivity small-animal SPECT/CT with submillimeter spatial resolution. Eur J Nucl Med Mol Imaging. 2006;33:S117. 